\title{
Suppressing the Effect of Impulsive Noise on Millimeter-Wave Communications Systems
}

\author{
Lara SHHAB ${ }^{1}$, Ahmet RIZANER ${ }^{2}$, Ali Hakan ULUSOY ${ }^{2}$, Hasan AMCA ${ }^{1}$ \\ ${ }^{1}$ Dept. of Electrical and Electronic Engineering, Faculty of Engineering, Eastern Mediterranean University, Famagusta, \\ North Cyprus via Mersin 10, Turkey \\ ${ }^{2}$ Dept. of Information Technology, School of Computing and Technology, Eastern Mediterranean University, Famagusta, \\ North Cyprus via Mersin 10, Turkey
}

shehablara@yahoo.com, \{ahmet.rizaner, alihakan.ulusoy, hasan.amca\}@emu.edu.tr

Submitted November 5, 2019 / Accepted March 3, 2020

\begin{abstract}
The Fifth Generation (5G) wireless communications systems are expected to satisfy higher data rates, network scalability, increased number of connections and higher traffic densities in a cost-effective manner. The key essence of $5 G$ technology resides in exploring the frequency bands at millimeter-Wave (mmWave) frequencies. As is well known, the presence of Impulsive Noise (IN) corrupts signals and leads to increased bit error rate and decreased spectral efficiency. In this paper, the performance of mmWave systems in multi-path fading channel and IN is studied and a new thresholding mechanism for the clipping and blanking filters to suppress the impulsive components of noise is suggested. The paper also presents the mathematical expressions to determine the optimum threshold selection for the filters. Simulation results show that use of clipping and blanking filters with the optimal threshold values reduces the adverse effect of IN and improves system performance significantly.
\end{abstract}

\section{Keywords}

Millimeter-wave, impulsive noise, clipping, blanking

\section{Introduction}

At the turn of the 20th century, wireless communication technology had started as one of the most important changes in human history that has been continuously shaping the way people live, work and communicate. Evolving from the First Generation (1G) to the Fourth Generation (4G) during the four decades enabled users to obtain, process, manage and disseminate information at a global scale. Wireless communication also enhanced human productivity in many areas of industry [1]. The growing demand to satisfy the traffic growth in the near future, pushed by the increasing demand for higher data rates and expanding capacities has led to the dawn of the Fifth Generation (5G) wireless communications systems [1-5]. $5 \mathrm{G}$ is expected to satisfy higher data rates, network scalability, higher number of connections and higher traffic densities accompanied by a significant reduction in service charges. Therefore, $5 \mathrm{G}$ improves network performance and increases capacity through cell splitting and increased number of base stations. However, the key feature of $5 \mathrm{G}$ technology resides in exploring the wide range of unused spectrum at extremely-high frequency-bands at millimeter-Wave (mmWave) frequencies [2]. The term millimeter-Wave covers the range of frequencies from $3 \mathrm{GHz}$ (where $\lambda=100 \mathrm{~mm}$ ) to $300 \mathrm{GHz}$ (where $\lambda=1 \mathrm{~mm}$ ). Hence, in the frequency range of $3 \mathrm{GHz}-300 \mathrm{GHz}$, the wavelengths vary between $100 \mathrm{~mm}-1 \mathrm{~mm}$, so it is referred as mmWave frequencies [3]. There are significant differences between the radio propagation characteristics of microwave and mmWave frequencies such as, the attenuation of mmWave frequencies through obstacles such as walls and ceilings are significantly higher than that of the microwave frequencies. These key differences in architectures and system performance of microwave and mmWave communications systems are presented in detail in [4].

The mmWave frequencies can be employed as a spectacular technology for the future $5 \mathrm{G}$ system to increase data rates significantly above those of the $3 \mathrm{G}$ and $4 \mathrm{G}$ systems [6]. Using high-frequencies between $30 \mathrm{GHz}$ and $300 \mathrm{GHz}$ could provide more bandwidth to deliver faster, higher-quality video and multimedia content as well as utilizing its large spectrum allocation to provide multi-gigabit communications services such as high definition TV and ultra-high definition video [7]. Several companies such as Samsung and Verizon performed their mmWave band tests at $28 \mathrm{GHz}$ and showed that this band is a viable band for small-cell cellular communications with propagation distances of over 200 meters [2], [8-10]. However, the use of such high frequencies as mmWave bands bears major drawbacks in terms of severe signal attenuation and blocking through the obstacles that makes the process of perceiving the vision of the cellular network a challenging task. The large signal attenuation and severe blocking by obstacles at mmWave frequencies can be counterfeit by employing many miniaturized antennas to be placed in small dimensions 
due to the very small wavelengths involved $[5,11,12]$. The engaged multiantenna systems can direct electrical energy into the intended points. Large number of antennas packed together at the base station and in the skin of cell phones or even within a chip produces very high gains. Nevertheless, using such multi-antenna systems in mmWave frequencies lead up to a noise behavior that could affect reliability of the communication channel and deteriorate its performance [13].

The proposed work assumes, based on previous research published in the literature that, Impulsive Noise (IN) present at the mmWave frequencies degrades communications quality. The common sources of IN could be man-made interference noise such as PAM signals or natural noise with impulsive behavior. Atmospheric and solar static signals due to thunderstorms and sunspots can also result in IN which extends into the mmWave frequency bands [14]. The estimation of IN in electricity subsystem at $6 \mathrm{GHz}$ band was investigated in [15] while the authors in [16] analyzed the performance of GPS receivers subject to IN at $10 \mathrm{GHz}$. The performance of 64-QAM signals in the hybrid AM-VSB/QAM optical fiber transmission system at $12 \mathrm{GHz}$ was researched in [17]. The Bit Error Rate (BER) performance of 16-QAM signals in an AM/16-QAM hybrid optical transmission system at $20 \mathrm{GHz}$ was estimated based on the Middleton class-A model in [18] and the work in [19] reported a $160 \mathrm{Gbit} / \mathrm{s} \mathrm{clock}$ recovery with an electro-optical PLL subject to IN at $40 \mathrm{GHz}$. ITU recommendation ITU-R P.372-8 also verified that for IN well above $1 \mathrm{GHz}$, the noise figure values are quite low and only the higher magnitude pulses appear above the receiver's noise threshold [20]. It has been shown in [21] that IN could degrade the performance of beamformers at high frequencies and new adaptive beamforming has been proposed that showed strong resistance to heavy-tailed distribution noises. In many physical radar environments, the performance of the applications such as airborne related to air-to-ground military radars such as mmWave seekers $(94 \mathrm{GHz})$ and obstacle detection radars $(35 \mathrm{GHz})$ mounted on helicopters, and automotive applications related to mmWave seekers car radars $(77 \mathrm{GHz})$ and traffic monitoring radars $(24 \mathrm{GHz})$ are affected negatively by the presence of IN [22].

Several methods have been investigated in the literature to mitigate the detrimental effects of IN on communications systems [23-26]. One of these methods used a nonlinear preprocessor at the receiver [23]. Clipping and blanking are considered to be the simple types of these preprocessors where a precoding matrix is used to enhance the performance of blanking at the transmitter. It also reduces the false alarms in detecting IN and improves Signal to Noise Ratio (SNR) [27]. The probability of missed clipping/blanking was minimized to enhance the performance of the system by the researchers in [28-30]. Due to its nonlinearity, blanking preprocessor performance could be corrupted by the harmful effects of its internal interference. As a result, an enhanced version was proposed to cope with such effects, achieving 1-4 dB SNR gain for variant impulsive environments [31]. The core of the proposed adaptive technique proposed in [32] was to find the appropriate method for reducing IN, based on using a level detector with a set of thresholds. The technique offered an improved BER performance compared to conventional techniques. Constructing a concept of Orthogonal Frequency-Division Multiplexing (OFDM) receiver capable of handling many kinds of impulsive interference could be verified using the adaptive calculation of the blanking threshold. In [33], an iterative structure that included blanking nonlinearity besides frequency-selective blanking nonlinearity enabled the proposed technique to be applicable for a wide range of OFDM systems. Despite the additional computational complexity of the iterative structure, information regarding the interference characteristics was not required. Some simple techniques for acquiring quasi-optimal thresholds for blanking and clipping preprocessors revolved on a structure of periodic pulse train function and Fourier series. They could verify a matching between the ideal design of optimal thresholds and the error performance for the proposed nonlinearity preprocessors [34]. The median of received signal samples in the OFDM receiver determined the clipping level for signal values. When compared to other schemes, the method introduced in [35] showed a better performance in maximizing SNR and minimizing the symbol error rate values. In [36], the authors conducted an approach without exploiting IN statistics for deriving and formulating the clipping threshold as a pairwise error probability optimization problem to suppress the error floor. It emulated the Maximum Likelihood decoder performance at high SNR under the Bernoulli Gaussian noise model with a wide range of parameters. However, [37] sought for obtaining the best clipping threshold by using the density evolution tool. It used the Cauchy distribution to determine clipping de-mapper slope, otherwise, the clipping de-mapper gained a satisfactory performance. The methods employed in the literature so far relied on using blanking/clipping preprocessors for IN suppression in power line communications and multiple input multiple output systems. None of these works was focused on mmWave communications systems. In fact, researches on mmWave communications systems have been mainly conducted under Gaussian noise and the effect of IN is mainly ignored $[6,7,11,38-40]$. A preliminary investigation of the effect of IN on mmWave communications systems using nonlinear preprocessor filters has been presented in [41]. Thus, a further study on reducing the effect of IN on mmWave communications systems needs to be done.

In this study, we employed blanking and clipping filters for reducing the effect of IN on mmWave communications systems. Since the existing thresholding mechanisms are mostly developed for microwave communications systems, they are not suitable for mmWave communications systems in their original form. In addition, the use of fixed threshold value does not promise optimal performance at different noise levels. Thus, a novel thresholding mechanism for IN mitigation is suggested and mathematical expressions for optimum threshold selection are obtained for the preprocessor filters. The optimal thresholding expression is derived by 
investigating the system performance under different scenarios to minimize the BER of the system. The effect of IN on BER and spectral efficiency of the system is examined with the use of optimal threshold values at the filters. As well as minimizing the BER, use of the proposed thresholding mechanism also maximizes the spectral efficiency of the system under IN.

The main structure of this paper is as follows. Section 1 describes the problem details and introduces the details of the previous research and their findings while Section 2 describes the mmWave communications system model. Section 3 presents the details of IN suppressing filters. Section 4 discusses simulation modeling and summarizes the results while Section 5 draws major conclusions and suggests some probable future work.

\section{System Model}

A common mmWave system receiver has a configuration where the array signal processing is partitioned into Radio Frequency (RF) combiners followed by baseband combiners. The RF precoder and combiner monitors the phases of the signals that pass through pure analog phase shifters into and out of the antenna elements to produce multiple beams in the direction of the dominant paths in the mmWave field. Every antenna element at the Base Station (BS) involves one RF chain, in which an RF chain includes a low-noise amplifier, downconverter and a digital to analog converter. The baseband precoder, meanwhile, provides an additional level of flexibility over the constant-gain/phase-only operations at the RF precoder. The precoders used in the article are $\mathbf{F}_{\mathrm{RF}}$ and $\mathbf{F}_{\mathrm{BB}}$ which are built by BS to approximate the dominant singular vectors of the channel. Whereas the combiners $\mathbf{W}_{\mathrm{RF}}$ and $\mathbf{W}_{\mathrm{BB}}$ are built in Mobile Station (MS) or the receiver.

The simplified block diagram of the proposed receiver model of the downlink mmWave communications system with large antenna arrays at MS is shown in Fig. 1. In this model, a BS with $N_{\mathrm{BS}}$ antennas and $N_{\mathrm{RF}}$ RF chains communicates with an MS having $N_{\mathrm{MS}}$ antennas and $N_{\mathrm{RF}} \mathrm{RF}$ chains.

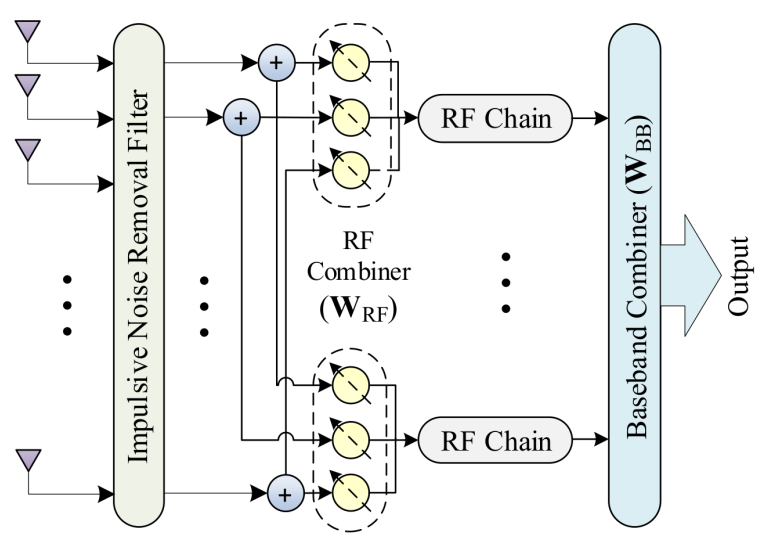

Fig. 1. Simplified BS receiver block diagram of mmWave system.
BS communicates with MSs via $N_{\mathrm{S}}$ data streams as described in [42]. An $N_{\mathrm{RF}} \times N_{\mathrm{S}}$ baseband precoder, $\mathbf{F}_{\mathrm{BB}}$ is assumed to be applied at BS followed by an $N_{\mathrm{BS}} \times N_{\mathrm{RF}} \mathrm{RF}$ precoder, $\mathbf{F}_{\mathrm{RF}}$. The combined hyrbrid BS precoding matrix, $\mathbf{F}_{\mathrm{T}}=\mathbf{F}_{\mathrm{RF}} \mathbf{F}_{\mathrm{BB}}$ with dimensions $N_{\mathrm{BS}} \times N_{\mathrm{S}}$ is formed from $\mathbf{F}_{\mathrm{RF}}$ and $\mathbf{F}_{\mathrm{BB}}$ to yield a discrete-time transmitted signal:

$$
\mathbf{x}=\mathbf{F}_{\mathrm{T}} \mathbf{S}
$$

where $\mathbf{s}$ is the transmitted symbol vector of dimension $N_{\mathrm{S}} \times 1$. The constant modulus entries of $\mathbf{F}_{\mathrm{RF}}$ implemented using analog phase shifters satisfies $\left|\left[\mathbf{F}_{\mathrm{RF}}\right]_{m, n}\right|^{2}=N_{\mathrm{BS}}^{-1}$, where $\left|\left[\mathbf{F}_{\mathrm{RF}}\right]_{m . n}\right|$ denotes the magnitude of the $(m, n)$ th element of $\mathbf{F}_{\mathrm{RF}}$. The received signal at the output of the narrowband block-fading channel that would be observed by MS is then:

$$
\mathbf{r}=\mathbf{H F}_{\mathrm{T}} \mathbf{S}+\mathbf{n}
$$

where $\mathbf{H}$ is the mmWave channel matrix of $N_{\mathrm{MS}} \times N_{\mathrm{BS}}$ elements between MS and BS as defined in [42-44], and $\mathbf{n}$ is the overall noise corrupting the received signal. The discretetime narrowband channel $\mathbf{H}$ with $L$ paths can be written as:

$$
\mathbf{H}=\sqrt{\frac{N_{\mathrm{BS}} N_{\mathrm{MS}}}{\rho}} \sum_{l=1}^{L} \alpha_{l} \mathbf{a}_{\mathrm{MS}}\left(\theta_{l}\right) \mathbf{a}_{\mathrm{BS}}^{H}\left(\phi_{l}\right)
$$

where $\rho$ denotes the average path-loss between BS and MS and $\alpha_{l}$ is the complex gain of the $l$ th propagation path. The variables $\theta_{l} \in[0,2 \pi]$ and $\phi_{l} \in[0,2 \pi]$ are the $l$ th path's angels of arrival and departure respectively. The vectors $\mathbf{a}_{\mathrm{MS}}$ and $\mathbf{a}_{\mathrm{BS}}$ represent the normalized received and transmit response vectors at an angle of $\theta_{l}$ and $\phi_{l}$ respectively.

In this system, we employed Uniform Linear Array (ULA) antenna architecture. This architecture is a collection of sensor elements such as dipole antennas, spaced along a straight line by a distance equal or less than the half of the wavelength. ULA antenna structure is used to improve SNR and gain in a particular direction. Assigning one RF chain to each antenna consumes a huge amount of power, thus usually hybrid beamforming is used with a limited number of RF chains to reduce the cost and power consumption.

The received signal $\mathbf{r}$ can then be obtained by processing the signal at MS by $\mathbf{W}_{\mathrm{T}}=\mathbf{W}_{\mathrm{RF}} \mathbf{W}_{\mathrm{BB}}$ as:

$$
\mathbf{y}=\mathbf{W}_{\mathrm{T}}^{H} \mathbf{H} \mathbf{F}_{\mathrm{T}} \mathbf{s}+\mathbf{W}_{\mathrm{T}}^{H} \mathbf{n}
$$

where $\mathbf{W}_{\mathrm{T}}$ is composed of RF and basedband combiners $\mathbf{W}_{\mathrm{RF}}$ and $\mathbf{W}_{\mathrm{BB}}$ respectively. In (4), $\mathbf{n}$ is modelled by Gaussian mixture model which is an approximation of the Middleton class-A noise model [45] that can be written as:

$$
\mathbf{n}=\mathbf{w}+\mathbf{z}
$$

where w represents the complex Additive White Gaussian Noise (AWGN) which occurs with a probability of $1-\epsilon$ and $\mathbf{z}$ represents IN which occurs with a probability of $\epsilon$. Assuming that the Gaussian terms are denoted as $N\left(0, \sigma_{\mathrm{w}}^{2}\right)$ and $N\left(0, \sigma_{\mathrm{z}}^{2}\right)$ the mixture distribution of the total noise has the following form: 


$$
f=(1-\epsilon) N\left(0, \sigma_{\mathrm{w}}^{2}\right)+\epsilon N\left(0, \sigma_{\mathrm{z}}^{2}\right)
$$

where $N($.$) is the Gaussian probability density function, \sigma_{\mathrm{w}}^{2}$ and $\sigma_{\mathrm{z}}^{2}$ denote the variances of AWGN and IN, which are related to the input SNR and Signal to Impulsive Noise Ratio (SINR), respectively.

\section{Impulsive Noise Removal Filters}

Assuming that, the signal amplitudes are considerably lower than the amplitudes of IN, as simple and efficient methods, blanking and clipping techniques can be applied to reduce the hazardous effects of IN in mmWave communications systems. However, improving the performance of the system is based on the convenient threshold value that corresponds to signal status. The signal amplitudes higher than the threshold value are assumed to be affected by IN, hence they need to be modified by the proposed techniques. While the threshold values are assigned to the affected values in clipping, the impulsive components are zeroed by blanking filter. In front of the receiver, the received signal $\mathbf{r}$, represented by (2) is passed through IN suppressing filter with blanking or clipping functions as shown in Fig. 2.

At the filter, the absolute values of the elements of the received signal vector are normalized by dividing them with the norm of the received signal vector $\mathbf{r}$ as:

$$
r_{\mathrm{N}}(i)=|r(i)| /\|\mathbf{r}\|
$$

where $|r(i)|$ represents the absolute value of the $i$ th component of the vector $\mathbf{r}$, and $\|\mathbf{r}\|$ denotes the norm of vector $\mathbf{r}$. The noise suppressing filter is used to detect the amplitude and the phase of the received signal to yield the filtered received signal by modifying the amplitude while keeping the phase unchanged.

In order to optimize system performance, the filter thresholds need to be selected carefully. With the choice of a proper threshold and multiplying the received signal with the combiner matrix $\mathbf{W}_{\mathrm{T}}^{H}$, the received signal at the combiner output $\left(\mathbf{y}_{\mathrm{f}}\right)$ becomes [42]:

$$
\mathbf{y}_{\mathrm{f}}=\mathbf{W}_{\mathrm{T}}^{H} \mathbf{r}_{\mathrm{f}}
$$

where $\mathbf{r}_{\mathrm{f}}$ is the filtered received signal.

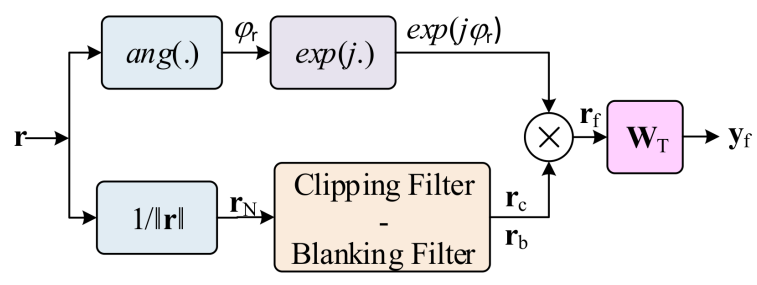

Fig. 2. Block diagram of the nonlinear preprocessor at the receiver.

\subsection{Clipping Filter}

If the amplitude of the $i$ th element of normalized received signal vector $r_{\mathrm{N}}(i)$ is greater than the clipping threshold, the output of the clipping filter is limited, otherwise, it is left unattended as in (9):

$$
r_{\mathrm{c}}(i)= \begin{cases}T_{\mathrm{c}}\|\mathbf{r}\| & r_{\mathrm{N}}(i)>T_{\mathrm{c}} \\ r(i) & r_{\mathrm{N}}(i) \leq T_{\mathrm{c}}\end{cases}
$$

where $T_{\mathrm{c}}$ is the clipping threshold. Then, the filtered received signal $\mathbf{r}_{\mathrm{f}}$ is constructed as:

$$
\mathbf{r}_{\mathrm{f}}=\mathbf{r}_{\mathrm{c}} \mathrm{e}^{-\mathrm{j} \varphi_{\mathrm{r}}}
$$

where $\varphi_{\mathrm{r}}$ is the phase angle of the received signal vector $\mathbf{r}$.

\subsection{Blanking Filter}

Whenever the amplitude of the $i$ th element of the normalized received signal vector, $r_{\mathrm{N}}(i)$, is greater than the blanking threshold, the output of the blanking filter is set to zero, otherwise, it is left unattended as in (11):

$$
r_{\mathrm{N}}(i)= \begin{cases}0 & r_{\mathrm{N}}(i)>T_{\mathrm{b}} \\ r(i) & r_{\mathrm{N}}(i) \leq T_{\mathrm{b}}\end{cases}
$$

where $T_{\mathrm{b}}$ is the blanking threshold. Then, the filtered received signal $\mathbf{r}_{\mathrm{f}}$ is constructed as:

$$
\mathbf{r}_{\mathrm{f}}=\mathbf{r}_{\mathrm{b}} \mathrm{e}^{-\mathrm{j} \varphi_{\mathrm{r}}}
$$

\section{Results and Discussion}

To assess the performance of the system, the downlink mmWave communications system shown in Fig. 1 is simulated in MATLAB with $N_{\mathrm{BS}}=64$ antennas and $16 \mathrm{RF}$ chains at BS communicating with $N_{\mathrm{MS}}=32$ antennas and $8 \mathrm{RF}$ chains at MS. Uniform linear array model is employed for the antenna arrays where the antennas are separated by half-wavelength distances. We consider the channel model defined in (3) with $L=3$ paths whose amplitudes are assumed to be Rayleigh distributed with an average power gain of 1 and path-loss exponent of 3. Although the system is designed to operate between $3-300 \mathrm{GHz}$ mmWave frequency band, it is tested at $28 \mathrm{GHz}$ carrier frequency with a bandwidth of $100 \mathrm{MHz}$. The hybrid precoding and combiner matrices are constructed as defined in [41] using the structure of a multi-resolution codebook with a resolution parameter of 192 having 2 beamforming vectors in each subset. The distance between the transmitter and the receiver is set to 50 meters throughout the simulations. It is assumed that both BS and MS have the channel knowledge. The simulation parameters are set for the signaling as 16-QAM and for IN channels SINR is kept constant as $-10 \mathrm{~dB}$. Each simulation scenario is repeated 1000 times to achieve an average performance in every setup. 


\subsection{Choice of Threshold Values}

In this section, we first show the effect of IN on the assumed mmWave communications system and investigate the importance of proper threshold selection by extensive simulations. A mathematical expression for the optimal threshold values is also proposed based on the experimental data obtained.

The first simulation is conducted to show the degradation effect of the IN on the BER performance. The performance of the mmWave communications system under Gaussian noise plus IN is compared in Fig. 3. It is obvious that IN degrades the functionality of the system gradually by increasing its density, $\epsilon$. The comparative values of SNR and the Effective SNR (SNRE), defined as the average signal power over the average total noise power at different $\epsilon$, are given in Tab. 1 when SINR equals to $-10 \mathrm{~dB}$. As seen from Tab. 1 , the presence of the impulsive components in the noise reduces the SNRE at the receiver significantly. Hence an effective mechanism to compensate for the effect of the IN component and increasing the effective SNR is needed.

The next simulations are performed to investigate the effect of threshold on BER and determine an optimal value of the threshold for blanking and clipping under IN. Selecting the optimum threshold value ensures suppressing IN while not removing useful signal content along with IN. Figure 4 shows how BER changes with different blanking thresholds under IN at $\epsilon=0.02$ and $\epsilon=0.04$ for different SNR values. It is shown that for every SNR, there is an ideal threshold that minimizes BER. When $\epsilon=0.02$, the minimum BER value approaches to $1 \times 10^{-3}$ when $T_{\mathrm{b}}$ equals 0.3 at $20 \mathrm{~dB}$ SNR. On the other side, when IN is more severe $(\epsilon=0.04)$, BER is about $6 \times 10^{-2}$ at $T_{\mathrm{b}}=0.25$ and $20 \mathrm{~dB}$ SNR. Figure 5 similarly presents BER against clipping thresholds under IN. The best BER value is obtained as $7 \times 10^{-2}$ when $T_{\mathrm{c}}=0.2$ and $\epsilon=0.02$ at $20 \mathrm{~dB}$ SNR. Nonetheless, the BER is about $2 \times 10^{-2}$ when $\epsilon=0.04$ with $T_{\mathrm{c}}=0.18$ at $20 \mathrm{~dB}$ SNR. As clearly observed from the results presented in Fig. 4 and Fig. 5, the best performance is achieved with the filters at a certain threshold value.

\begin{tabular}{|c|c|c|c|c|c|c|}
\hline & \multicolumn{7}{|c|}{ SNRE [dB] } \\
\hline SNR [dB] & $\epsilon=0.00$ & 0.01 & 0.02 & 0.03 & 0.04 & 0.05 \\
\hline-2.00 & -2.00 & -2.22 & -2.44 & -2.64 & -2.84 & -3.02 \\
0.00 & 0.00 & -0.37 & -0.72 & -1.04 & -1.34 & -1.61 \\
2.00 & 2.00 & 1.40 & 0.87 & 0.40 & -0.02 & -0.41 \\
4.00 & 4.00 & 3.06 & 2.29 & 1.64 & 1.07 & 0.56 \\
6.00 & 6.00 & 4.58 & 3.51 & 2.65 & 1.93 & 1.32 \\
8.00 & 8.00 & 5.90 & 4.49 & 3.43 & 2.58 & 1.87 \\
10.00 & 10.00 & 7.01 & 5.26 & 4.01 & 3.05 & 2.25 \\
12.00 & 12.00 & 7.89 & 5.82 & 4.42 & 3.37 & 2.52 \\
14.00 & 14.00 & 8.56 & 6.22 & 4.70 & 3.58 & 2.69 \\
16.00 & 16.00 & 9.04 & 6.49 & 4.89 & 3.73 & 2.81 \\
18.00 & 18.00 & 9.37 & 6.66 & 5.01 & 3.82 & 2.88 \\
20.00 & 20.00 & 9.59 & 6.78 & 5.09 & 3.88 & 2.93 \\
22.00 & 22.00 & 9.74 & 6.86 & 5.14 & 3.91 & 2.96 \\
\hline
\end{tabular}

Tab. 1. SNRE versus SNR at different $\epsilon$ values.

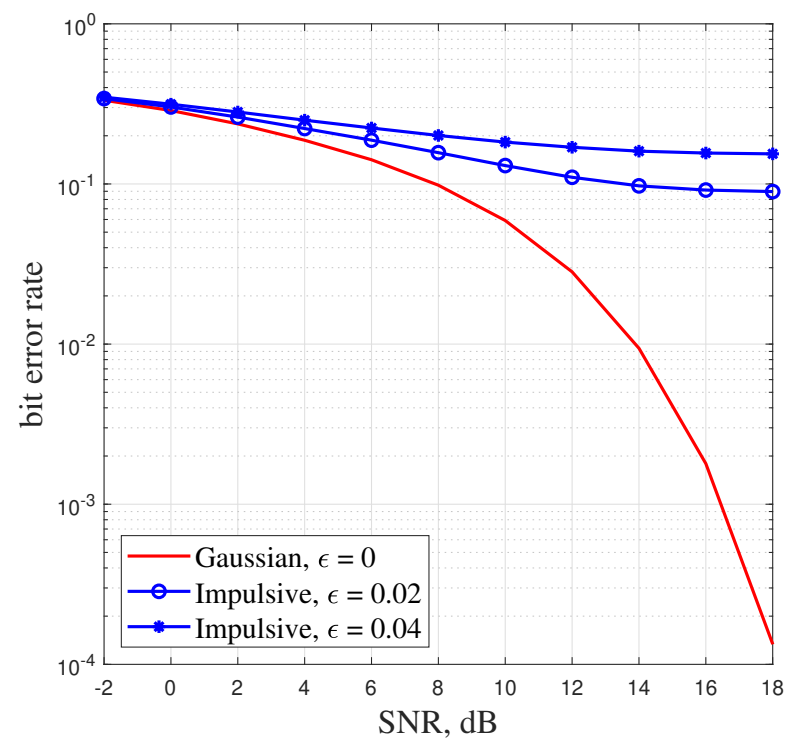

Fig. 3. BER of mmWave system versus SNR under Gaussian $(\epsilon=0)$ and IN $(\epsilon=0.02, \epsilon=0.04)$.
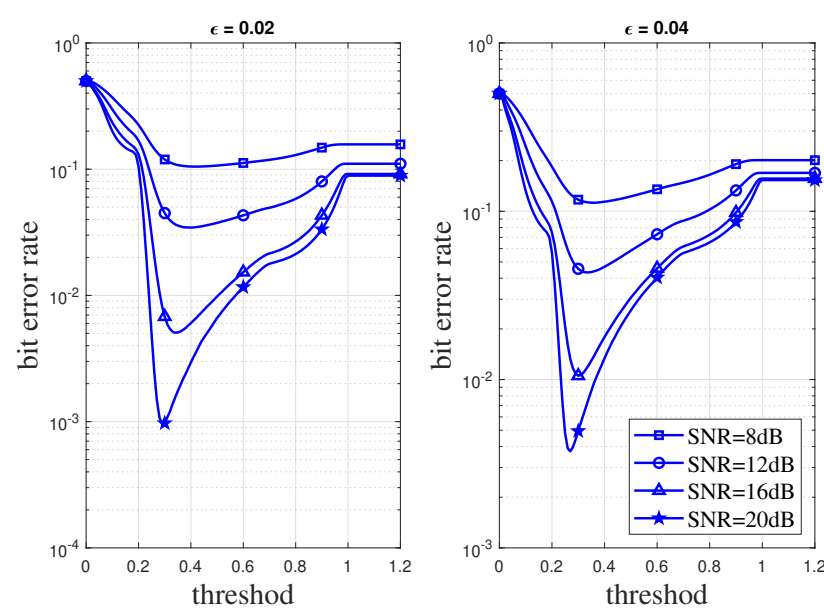

Fig. 4. BER against blanking threshold under IN $(\epsilon=0.02$, $\epsilon=0.04)$.
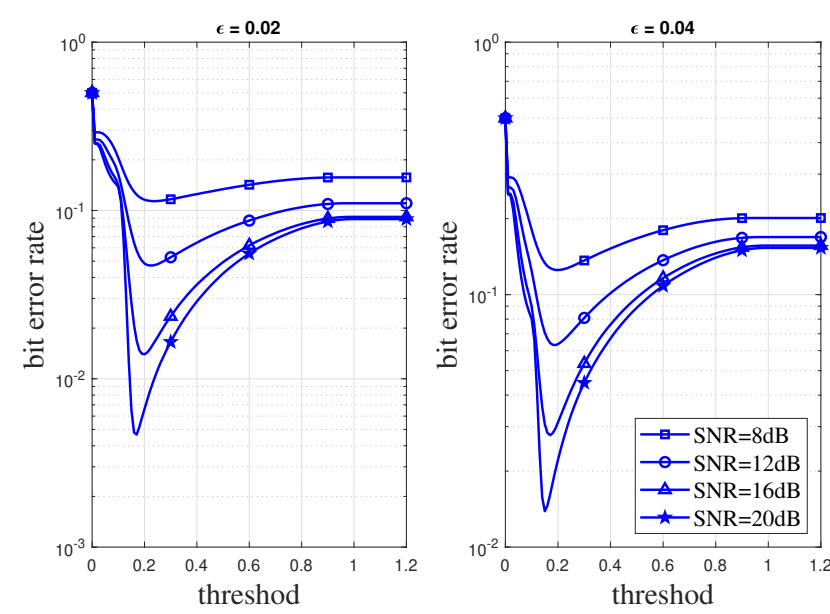

Fig. 5. BER against clipping threshold under IN $(\epsilon=0.02$, $\epsilon=0.04)$. 

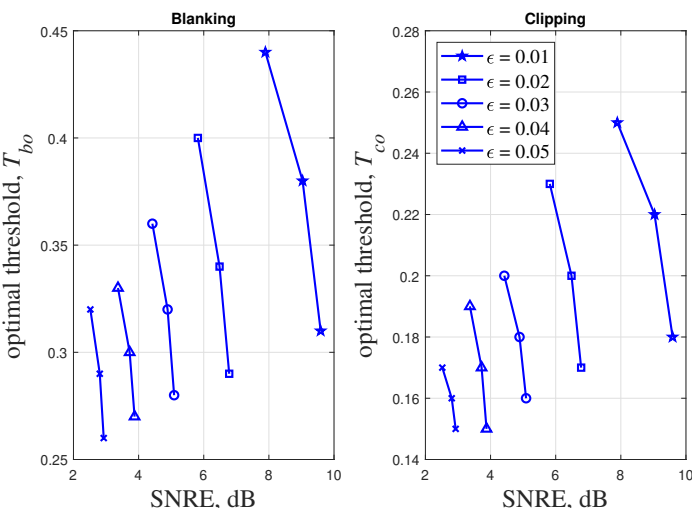

Fig. 6. Optimal blanking/clipping threshold versus SNRE.

Figure 6 presents how the optimal threshold values for blanking and clipping change with SNRE at different $\epsilon$ values respectively. An analytical expression for the optimal threshold is obtained by fitting the data collected, as presented in Fig. 6, to a polynomial surface of degree 2 in SNRE and $\epsilon$ as:

$$
T_{\mathrm{o}}\left(\gamma_{\mathrm{e}}, \epsilon\right)=a_{0}+a_{1} \gamma_{\mathrm{e}}+a_{2} \epsilon+a_{3} \gamma_{\mathrm{e}}^{2}+a_{4} \gamma_{\mathrm{e}} \epsilon+a_{5} \epsilon^{2}
$$

where $a_{0}=0.8279, a_{1}=0.002594, a_{2}=0.6283, a_{3}=$ $-0.002743, a_{4}=-2.943, a_{5}=-59.954$ for optimal threshold for blanking filter $\left(T_{\mathrm{bo}}\right)$ and $a_{0}=0.5525, a_{1}=-0.01492$, $a_{2}=-3.886, a_{3}=-0.0007977, a_{4}=-1.169, a_{5}=4.239$ for the optimal threshold of clipping filter $\left(T_{\mathrm{co}}\right) \cdot \gamma_{\mathrm{e}}$ is used to represent SNRE in (13) and can be calculated as:

$$
\gamma_{\mathrm{e}}=10 \log _{10}\left(\frac{P_{\mathrm{av}}}{\sigma_{\mathrm{e}}}\right)
$$

where $P_{\mathrm{av}}$ stands for average received power, and $\sigma_{\mathrm{e}}=$ $(1-\epsilon) \sigma_{\mathrm{w}}^{2}+\epsilon \sigma_{\mathrm{z}}^{2}$.

\subsection{BER Performance Improvement with the Use of Blanking/Clipping Filter}

The BER performance improvement with the use of the proposed blanking and clipping filters is presented in this section. For all of the simulations presented in this and the following sections, the optimal threshold values for blanking and clipping filters are obtained from (13).

Figures 7 and 8 present the performance of the proposed system with the optimal threshold values under IN at $\epsilon=0.02$ and 0.04 respectively. The results show that the performance of the proposed system with filters under IN significantly outperforms the performance of the system without filters at all SNRs since the effect of IN is reduced successfully by the filters. It is worth mentioning that the BER performance levels off at SNR values greater than $18 \mathrm{~dB}$ since the effective SNR is almost constant after that value as observed in Tab. 1. As seen from the results of Fig. 7 band Fig. 8, blanking filter performs better than the clipping filter.

Figure 9 illustrates BER versus $\epsilon$ for the proposed system at $16 \mathrm{~dB}$ and $20 \mathrm{~dB}$ SNR values with and without blanking and clipping filters. Both filters act efficiently to reduce the effect of IN, however, as anticipated, blanking overcomes clipping.

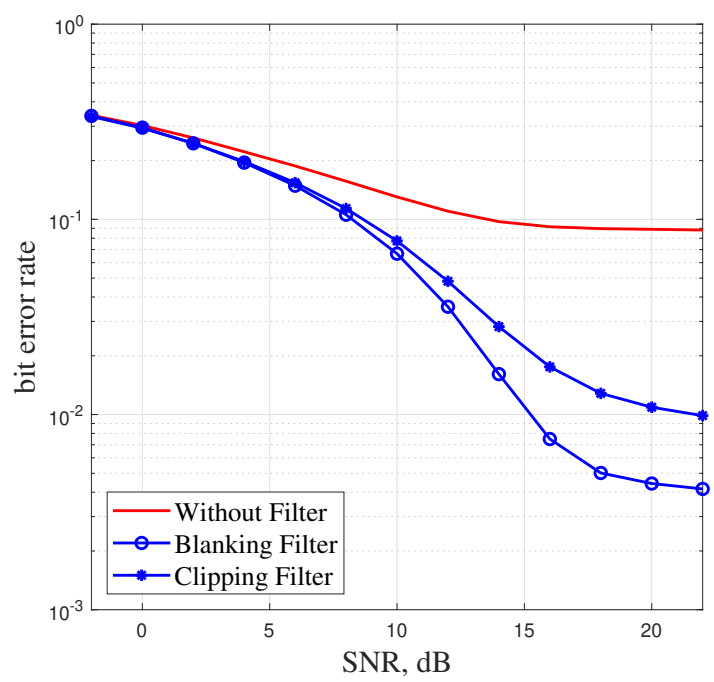

Fig. 7. BER of mmWave system with and without clipping and blanking filters versus SNR under IN $(\epsilon=0.02)$.

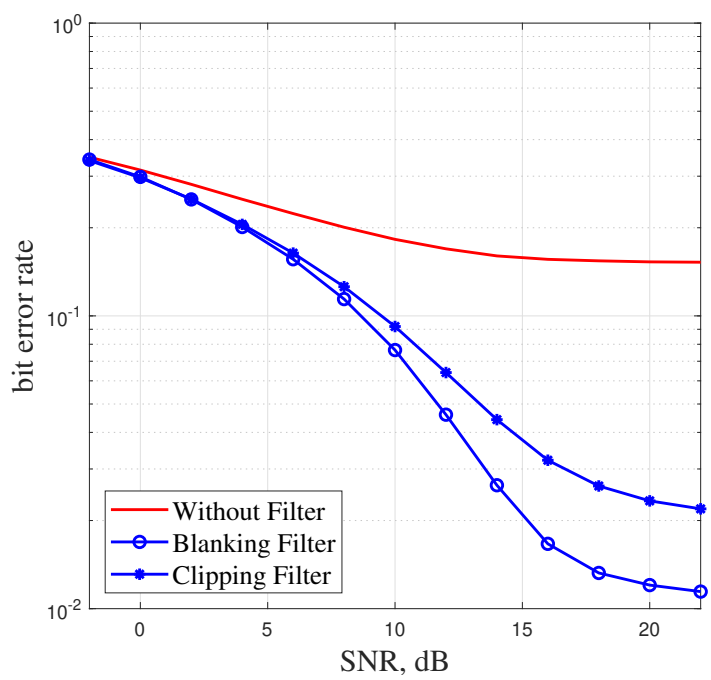

Fig. 8. BER of mmWave system with and without clipping and blanking filters versus SNR under IN $(\epsilon=0.04)$.

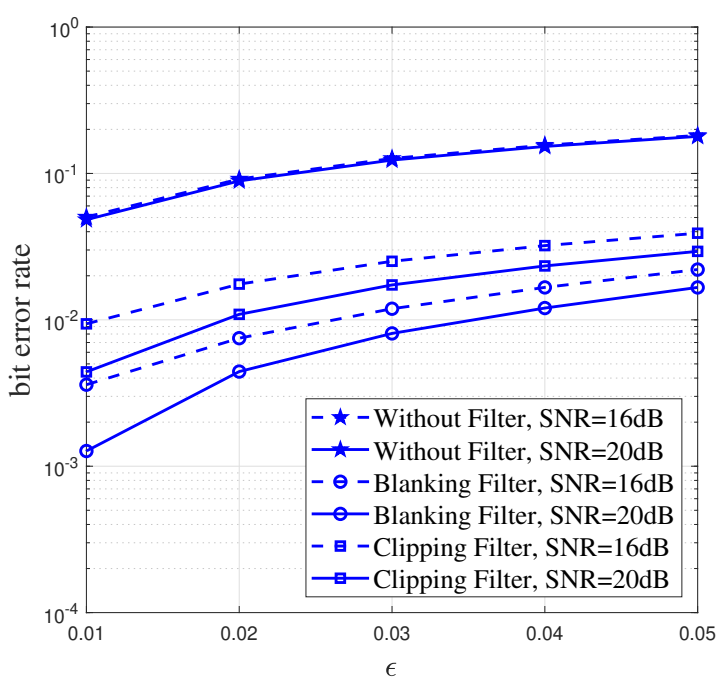

Fig. 9. BER of mmWave system with and without clipping and blanking filters versus $\epsilon$ under IN for SNR $=16$ and $20 \mathrm{~dB}$. 


\subsection{Spectral Efficiency Improvement with the Use of Blanking/Clipping Filter}

We show the effect of IN on the spectral efficiency of the system and the improvement in the spectral efficiency with the use of blanking and clipping filters. The spectral efficiency curves in this section are obtained from the following rate expression:

$$
\begin{aligned}
R=\log _{2} \mid \mathbf{I}_{N_{\mathrm{s}}}+ & \frac{P_{\mathrm{av}}}{\sigma_{\mathrm{e}} N_{\mathrm{S}}}\left(\mathbf{W}_{\mathrm{RF}} \mathbf{W}_{\mathrm{BB}}\right)^{H} \mathbf{H F}_{\mathrm{RF}} \mathbf{F}_{\mathrm{BB}} \\
& \times \mathbf{F}_{\mathrm{BB}}^{H} \mathbf{R}^{-1} \mathbf{F}_{\mathrm{BB}}^{H} \mathbf{H}^{H}\left(\mathbf{W}_{\mathrm{RF}} \mathbf{W}_{\mathrm{BB}}\right) \mid
\end{aligned}
$$

where, for a matrix || represents the determinant.

In Fig. 10, the spectral efficiency of the system is presented under Gaussian noise and IN at $\epsilon=0.02$ and 0.04 without applying the filters. It can be noticed from the given results that IN affects the spectral efficiency of the system negatively.

The effect of filters on the spectral efficiency is presented in Figs. 11 and 12. The results show that the spectral efficiency of the system is successfully improved by the use of filters under IN. Finally, the spectral efficiency of the system with and without the filters is presented for different $\epsilon$ values at several SNR values in Fig. 13. Again, it is seen that the use of a blanking filter gives better spectral efficiency performance than the clipping filter.

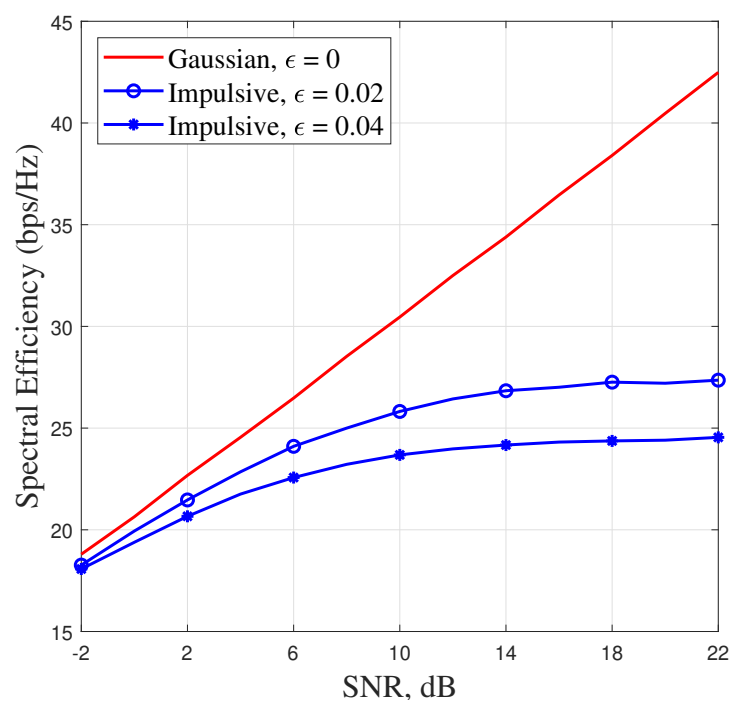

Fig. 10. Spectral efficiency of mmWave system versus SNR under Gaussian and IN.

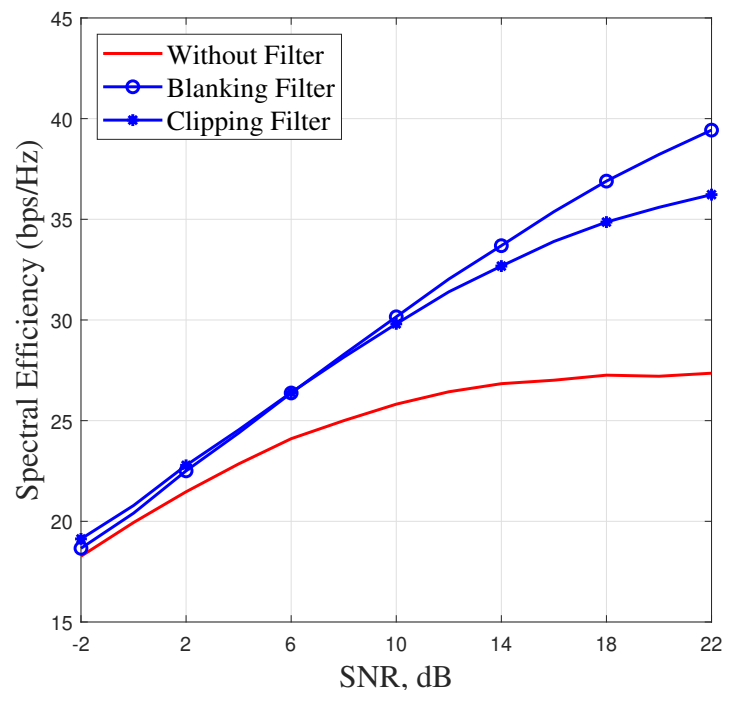

Fig. 11. Spectral efficiency of mmWave system under IN $(\epsilon=$ 0.02 ) with and without blanking and clipping filters.

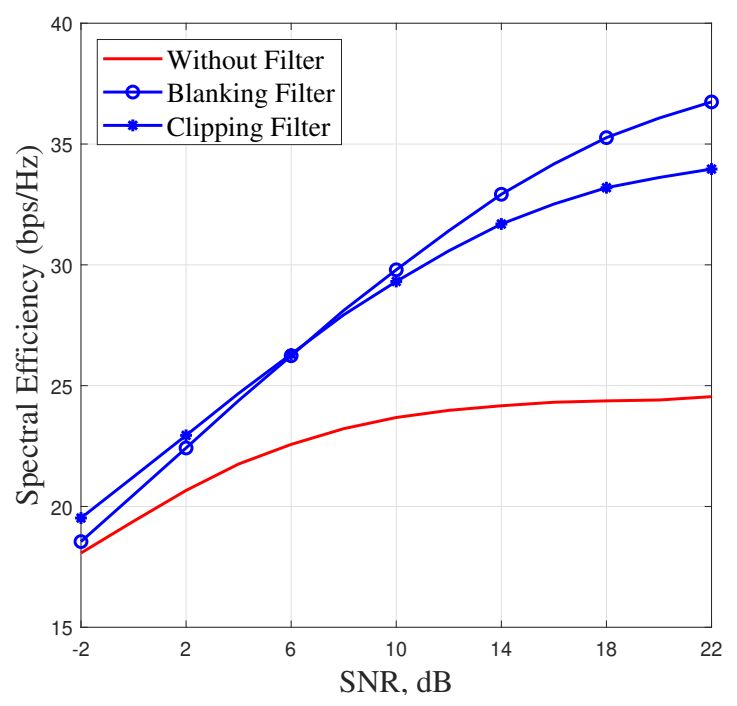

Fig. 12. Spectral efficiency of mmWave syste under IN $(\epsilon=$ 0.04 ) with and without blanking and clipping filters.

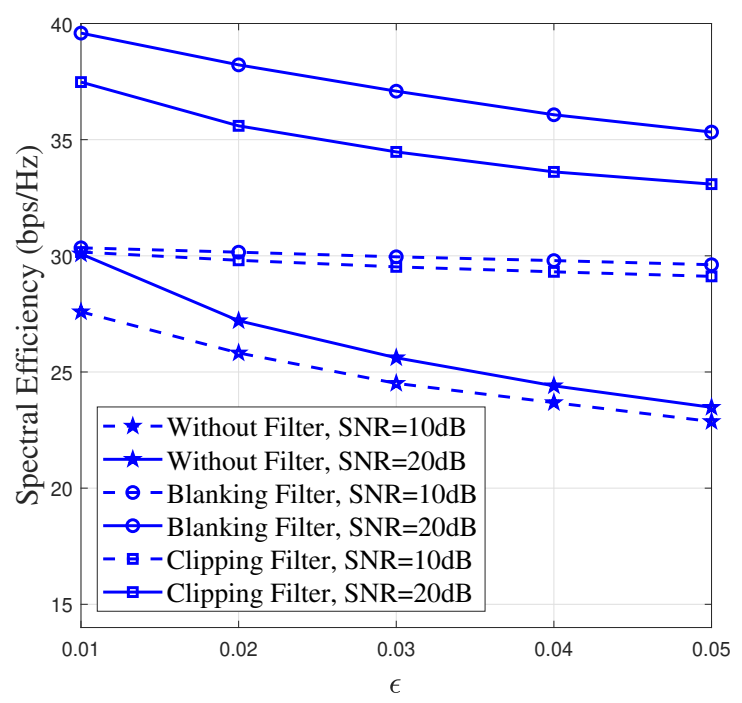

Fig. 13. Spectral efficiency of mmWave system versus $\epsilon$ under IN with and without blanking and clipping filters. 


\section{Conclusions}

The paper studies the use of clipping and blanking preprocessor filters to suppress the effect of IN on mmWave communications systems. It was shown by extensive simulation results that, choice of optimal threshold is crucial for the system performance when reducing the effect of impulsive components of the noise. Therefore, the paper also presents analytical expressions for determining the optimal threshold values for the proposed clipping and blanking filter architectures to minimize BER and maximize the spectral efficiency in IN. The numerical results have shown that the method involving the integration of blanking and clipping filters with the optimal threshold values can reduce the effect of IN and improve the system performance significantly. Although, the results have clearly demonstrated the effectiveness of both techniques to attain a significant enhancement in reducing IN in mmWave cellular system, the improvement obtained by blanking filters slightly outperforms that of the clipping filters.

\section{Acknowledgments}

We are thankful to the editor and anonymous referees for their constructive suggestions and comments.

\section{References}

[1] YUAN, Y., ZHAO, X. 5G vision, scenarios and enabling technologies. ZTE Communications, 2015, vol. 13, no. 1, p. 3-10. DOI: $10.3969 /$ j.issn.1673-5188.2015.01.001

[2] ROH, W., SEOL, J.Y., PARK, J. et al. Millimeter-wave beamforming as an enabling technology for $5 \mathrm{G}$ cellular communications: Theoretical feasibility and prototype results. IEEE Communications Magazine, 2014, vol. 52, no. 2, p. 106-113. DOI: 10.1109/MCOM.2014.6736750

[3] ALSHARIF, M.H., NORDIN, R. Evolution towards fifth generation $(5 \mathrm{G})$ wireless networks: Current trends and challenges in the deployment of millimetre wave, massive MIMO, and small cells. Telecommunication Systems, 2017, vol. 64, no. 4, p. 617-637. DOI: $10.1007 / \mathrm{s} 11235-016-0195-\mathrm{x}$

[4] SHAFI, M., ZHANG, J., THATRIA, H. et al. Microwave vs. millimeter-wave propagation channels: Key differences and impact on 5G cellular systems. IEEE Communications Magazine, 2018, vol. 56, no. 12, p. 14-20. DOI: 10.1109/MCOM.2018.1800255

[5] ALNOMAN, A., ANPALAGAN, A. Towards the fulfillment of 5G network requirements: Technologies and challenges. Telecommunication Systems, 2017, vol. 65, no. 1, p. 101-116. DOI: $10.1007 / \mathrm{s} 11235-016-0216-9$

[6] ROUFARSHBAF, H., MADHOW, U., RAJAGOPAL, S. OFDMbased analog multiband: A scalable design for indoor mmwave wireless communication. In IEEE Global Communications Conference (GLOBECOM). Austin (USA), 2014, p. 3267-3272. DOI: $10.1109 /$ GLOCOM.2014.7037310
[7] NIU, Y., LI, Y., JIN, D., et al. A survey of millimeter wave communications (mmWave) for 5G: Opportunities and challenges. Wireless Networks, 2015, vol. 21, no. 8, p. 2657-2676. DOI: $10.1007 / \mathrm{s} 11276-015-0942-\mathrm{z}$

[8] RAPPAPORT, T. S., MACCARTNEY, G. R., SAMIMI, M. K., et al. Wideband millimeter-wave propagation measurements and channel models for future wireless communication system design. IEEE Transactions on Communications, 2015, vol. 63, no. 9, p. 3029-3056. DOI: 10.1109/TCOMM.2015.2434384

[9] MACCARTNEY, G. R., RAPPAPORT, T. S., SUN, S., et al. Indoor office wideband millimeter-wave propagation measurements and channel models at 28 and $73 \mathrm{GHz}$ for ultra-dense $5 \mathrm{G}$ wireless networks. IEEE Access, 2015, vol. 3, p. 2388-2424. DOI: 10.1109/ACCESS.2015.2486778

[10] PÄTZOLD, M. 5G developments are in full swing. IEEE Vehicular Technology Magazine, 2017, vol. 12, no. 2, p. 4-12. DOI: 10.1109/MVT.2017.2681978

[11] AKDENIZ, M. R., LIU, Y., SAMIMI,M. K., et al. Millimeter wave channel modeling and cellular capacity evaluation. IEEE Journal on Selected Areas in Communications, 2014, vol. 32, no. 6 , p. 1164-1179. DOI: 10.1109/JSAC.2014.2328154

[12] PESHWE, P., KOTHARI, A. Performance enhancement of millimeter wave antenna with integrated inter-digital capacitor structure. Radioengineering, 2018, vol. 27, no. 3, p. 654-661. DOI: $10.13164 /$ re.2019.0439

[13] HUANG, K.C., WANG. Z., Millimeter Wave Communication Systems. Piscataway, (USA): John Wiley \& Sons, 2011. ISBN: 978-0470404621

[14] MIDDLETON, D. An Introduction to Statistical Communication Theory. Piscataway, (USA): Wiley-IEEE Press, 1996. ISBN: 978-0780311787

[15] SHAN, Q., GLOVER, I. A., ATKINSON, R. J., et al. Estimation of impulsive noise in an electricity substation. IEEE Transactions on Electromagnetic Compatibility, 2011, vol. 53, no. 3, p. 653-663. DOI: 10.1109/TEMC.2010.2092782

[16] LIU, L., AMIN, M. G., Performance analysis of GPS receivers in non-Gaussian noise incorporating precorrelation filter and sampling rate. IEEE Transactions on Signal Processing, 2008, vol. 56, no. 3 , p. 990-1004. DOI: 10.1109/TSP.2006.890827

[17] PHAM, K., CONRADI, J., CORMACK, G., et al. Impact of noise and nonlinear distortion due to clipping on the BER performance of a 64-QAM signal in hybrid AM-VSB/QAM optical fiber transmission system. Journal of Lightwave Technology, 1995, vol. 13, no. 11, p. 2197-2201. DOI: $10.1109 / 50.482039$

[18] MAEDA, K., NAKATA, H., FUJITO, K. Analysis of BER of 16QAM signal in AM/16QAM hybrid optical transmission system. Electronics Letters, 1993, vol. 29, no. 7, p. 640-642. DOI: 10.1049/el:19930428

[19] BOERNER, C., SCHUBERT, C., SCHMIDT, C., et al. 160 Gbit/s clock recovery with electro-optical PLL using bidirectionally operated electroabsorption modulator as phase comparator. Electronics Letters, 2003, vol. 39, no. 14, p. 1071-1073. DOI: $10.1049 / \mathrm{el}: 20030674$

[20] ITU-R. Telecommunications Union ITU-R Recommendation, Radio noise, P.372-8 (04/03), 2003, 75 pages. [Online] Cited 2019-11-01. Available at: https://www.itu.int/rec/R-REC-P.372-8-200304-S/en

[21] KATKOVNIK, V. A new concept of adaptive beamforming for moving sources and impulse noise environment. Signal Processing, 2000, vol. 80, no. 9, p. 1863-1882. DOI: 10.1016/S0165-1684(00)00094-3 
[22] LEE, M. S., KATKOVNIK, V., KIM, Y. H. Robust approximate median beamforming for phased array radar with antenna switching. Signal Processing, 2004, vol. 84, no. 9, p. 1667-1675. DOI: $10.1016 /$ j.sigpro.2004.05.007

[23] JUWONO, F.H., GUO, Q., HUANG, D., et al. Deep clipping for impulsive noise mitigation in OFDM-based power-line communications. IEEE Transactions on Power Delivery, 2014, vol. 29, no. 3, p. 1335-1343. DOI: 10.1109/TPWRD.2013.2294858

[24] ROZIC, N., BANELLI, P., BEGUSIC, D., et al. Multiple-threshold estimators for impulsive noise suppression in multicarrier communications. IEEE Transactions on Signal Processing, 2018, vol. 66, no. 6, p. 1619-1633. DOI: 10.1109/TSP.2018.2793895

[25] DUTTA, T., SATIJA, U., RAMKUMAR, B., et al. Blind impulse estimation and removal using sparse signal decomposition framework for OFDM systems. Circuits Systems and Signal Processing, 2018, vol. 37, no. 2, p. 847-861. DOI: 10.1007/s00034-017-0573-y

[26] RİZANER, A., ULUSOY, A. H., AMCA, H. Adaptive fuzzy assisted detector under impulsive noise for DVB-T systems. Optik - International Journal for Light and Electron Optics, 2016, vol. 127, no. 13, p. 5196-5199. DOI: 10.1016/j.ijleo.2016.02.079

[27] JUWONO, F. H., REINE, R., LIU, L., et al. Performance of impulsive noise blanking in precoded OFDM-based PLC systems. In IEEE International Conference on Communication Systems (ICCS). Shenzhen (China), 2016, p. 1-6. DOI: 10.1109/ICCS.2016.7833562

[28] RABIE, K. M., ALSUSA, E. Improved DPTE technique for impulsive noise mitigation over power-line communication channels. $A E U$ International Journal of Electronics and Communications, 2015, vol. 69, no. 12, p. 1847-1853. DOI: 10.1016/j.aeue.2015.09.012

[29] RABIE, K. M., ALSUSA, E. Performance analysis of adaptive hybrid nonlinear preprocessors for impulsive noise mitigation over power-line channels. In IEEE International Conference on Communications (ICC). London (UK), 2015, p. 728-733. DOI: 10.1109/ICC.2015.7248408

[30] RABIE, K., ALSUSA, E., FAMILUA, A., et al. Constant envelope OFDM transmission over impulsive noise power-line communication channels. In IEEE International Symposium on Power Line Communications and its Applications (ISPLC). Austin (USA), 2015, p. 13-18. DOI: 10.1109/ISPLC.2015.7147582

[31] SARABCHI, F., NERGUIZIAN, C. Impulsive noise mitigation for OFDM-based systems using enhanced blanking nonlinearity. In IEEE 25th Annual International Symposium on Personal, Indoor, and Mobile Radio Communication (PIMRC). Washington (USA), 2014, p. 841-845. DOI: 10.1109/PIMRC.2014.7136282

[32] HAKAM, A., KHALID, M., ALY, N. A., et al. MIMO-OFDM system with impulsive noise reduction technique based on auto level selection mechanism. In IEEE 15th International Conference on Communication Technology (ICCT). Guilin (China), 2013, p. 229-233. DOI: 10.1109/ICCT.2013.6820377

[33] EPPLE, U., SCHNELL, M. Advanced blanking nonlinearity for mitigating impulsive interference in OFDM systems. IEEE Transactions on Vehicular Technology, 2017, vol. 66, no. 1, p. 146-158. DOI: 10.1109/TVT.2016.2535374

[34] OH, H., NAM, H. Design and performance analysis of nonlinearity preprocessors in an impulsive noise environment. IEEE Transactions on Vehicular Technology, 2017, vol. 66, no. 1, p. 364-376. DOI: 10.1109/TVT.2016.2547889

[35] ALI, Z. Hybrid median-nulling scheme for impulsive noise mitigation in OFDM transmission. In IEEE Fourth International Conference on Aerospace Science and Engineering (ICASE). Islamabad (Pakistan), 2015, p. 1-5. DOI: 10.1109/ICASE.2015.7489518
[36] CHEN, C., MOW, W.H. Optimized metric clipping decoder design for impulsive noise channels at high signal-to-noise ratios. In IEEE 36th Sarnoff Symposium. Newark (USA), 2015, p. 46-49. DOI: 10.1109/SARNOF.2015.7324641

[37] MÂAD, H. B., GOUPIL, A., CLAVIER, L., et al. Robust clipping demapper for LDPC decoding in impulsive channel. In IEEE 6th International Symposium on IEEE Turbo Codes and Iterative Information Processing (ISTC). Brest (France), 2010, p. 231-235. DOI: $10.1109 /$ ISTC.2010.5613845

[38] ALKHATEEB, A., LEUS, G., HEATH, R. W. Limited feedback hybrid precoding for multi-user millimeter wave systems. IEEE Transactions on Wireless Communications, 2015, vol. 14, no. 11, p. 6481-6494. DOI: 10.1109/TWC.2015.2455980

[39] YU, X., SHEN, Y. J, ZHANG, J., et al. Alternating minimization algorithms for hybrid precoding in millimeter wave MIMO systems. IEEE Journal of Selected Topics in Signal Processing, 2016, vol. 10, no. 3, p. 485-500. DOI: 10.1109/JSTSP.2016.2523903

[40] XIE, Y., BO, L., ZHONGJIANG, Y, et.al. A general hybrid precoding method for mmWave massive MIMO systems. Radioengineering, 2019, vol. 28, no. 2, p. 439-446. DOI: 10.13164/re.2019.0439

[41] SHHAB, L. M. H., RIZANER, A., ULUSOY, A. H., et al. Impact of impulsive noise on millimeter wave cellular systems performance. In IEEE 10th UK-Europe-China Workshop on Millimetre Waves and Terahertz Technologies (UCMMT). Liverpool (UK), 2017, p. 1-4. DOI: 10.1109/UCMMT.2017.8068495

[42] ALKHATEEB, A., AYACH, O. E., LEUS, G., et al. Channel estimation and hybrid precoding for millimeter wave cellular systems. IEEE Journal of Selected Topics in Signal Processing, 2014, vol. 8, no. 5, p. 831-846. DOI: 10.1109/JSTSP.2014.2334278

[43] EL AYACH, O., RAJAGOPAL, S., ABU-SURRA, S., et al. Spatially sparse precoding in millimeter wave MIMO systems. IEEE Transactions on Wireless Communications, 2014, vol. 13, no. 3, p. 1499-1513. DOI: 10.1109/TWC.2014.011714.130846

[44] RAPPAPORT, T. S., HEATH JR., R. W., DANIELS, R. C., et al. Millimeter Wave Wireless Communications. London (UK): Pearson Education, 2014. ISBN: 978-0132172288

[45] WANG, X., POOR, H. V. Robust multiuser detection in non-Gaussian channels. IEEE Transactions on Signal Processing, 1999, vol. 47, no. 2, p. 289-305. DOI: 10.1109/78.740103

\section{About the Authors ...}

Lara SHHAB received the B.S. degree in Electrical Engineering from Jordan University of Science and Technology, Irbid, Jordan in 2002 and M.S. degrees in Computer and Communication Engineering from the Malaysian National University, Kuala Lumpur, Malaysia in 2005. She is currently pursuing the Ph.D. degree in Electrical and Electronic Engineering at Eastern Mediterranean University, North Cyprus, Turkey. From 2005 to 2007, she was a Full Time Lecturer at Granada College, Jordan, and from 2008 to 2011, she was a Full Time Lecturer at Intermediate College, Jordan. From 2011 to 2013, she was a Full Time Lecturer at Taibah University, Saudi Arabia. Her research interests include 5G technology, millimeter wave, and impulsive noise.

Ahmet RiZANER was born in Cyprus on January 21, 1974. He received the B.S., M.S. and Ph.D. degrees in Electrical and Electronic Engineering from the Eastern Mediterranean 
University (EMU), North Cyprus, in 1996, 1998 and 2004, respectively. He joined the department of Information Technology, EMU, in 2004 as an instructor where he became a Visiting Assistant Professor in 2005, an Assistant Professor in 2007, an Associate Professor in 2013 and a Professor in 2019. He served as the coordinator of the department of Information Technology between 2008 and 2013. Dr. Rizaner was appointed as the Vice Director of Institute of Graduate Studies and Research of EMU in 2015. His main research interests include wireless communication, adaptive channel estimation, fuzzy channel estimation, multiuser detection techniques, digital video broadcasting, mmWave communication and intrusion detection in mobile ad-hoc networks.

Ali Hakan ULUSOY was born in Eskişehir, Turkey, on June 3, 1974. He graduated from the double major program of department of Electrical and Electronic Engineering (EEE) and department of Physics in Eastern Mediterranean University (EMU), North Cyprus, in 1996. He received his M.S. and Ph.D. degrees in EEE, EMU in 1998 and 2004, respectively. Then, he joined department of Information Technology, EMU, in 2004 as an Instructor where he became a Visiting Assistant Professor in 2005, an Assistant Professor in 2007, an Associate Professor in 2013 and a Professor in 2019. He worked as the assistant director from January 2012 to August 2017 and the acting director from August 2017 to December 2019 of the Institute of Graduate Studies and Research (IGSR), EMU. He is currently working as the director of IGSR since January 2020. His research interests include wireless communications, receiver design, channel estimation, fuzzy systems, wireless networks, cloud computing, millimeter wave communications and healthcare system development.

Hasan AMCA received his B.S. degree in Electrical and Electronic Engineering from the Eastern Mediterranean University (formerly called Higher Technological Institute) in the North Cyprus in 1984, the M.S. degree from the University of Essex (UK) in 1985 and the Ph.D. degree from the University of Bradford (UK) in 1993. He worked as a Lecturer in the Electrical and Electronic Engineering Department of the EMU. He served as the Director of the School of Computing and Technology, the Dean of Engineering Faculty, Director of Continuing Education Center and Vice Rector responsible for International Relations and Recruitment at EMU. He also served as the chair of Board of Trustees of Ataturk Teachers Academy. Prof. Dr. Hasan AMCA is a senior member of the IEEE. Currently, he is member of the Information and Communication Technology Regulatory Board in North Cyprus. His current research interests are channel estimation and detection over multipath propagation medium for $3 \mathrm{G} / 4 \mathrm{G} / 5 \mathrm{G}$ mobile communications systems, OFDM, LTE, millimeterwave communications, digital video broadcasting and mobile payment systems. 